\title{
ELLIPTIC THEORY ON MANIFOLDS WITH CORNERS: II. HOMOTOPY CLASSIFICATION AND $K$-HOMOLOGY
}

\author{
VLADIMIR NAZAIKINSKII, ANTON SAVIN, AND BORIS STERNIN
}

\begin{abstract}
We establish the stable homotopy classification of elliptic pseudodifferential operators on manifolds with corners and show that the set of elliptic operators modulo stable homotopy is isomorphic to the $K$-homology group of some stratified manifold. By way of application, generalizations of some recent results due to Monthubert and Nistor are given.
\end{abstract}

\section{Contents}

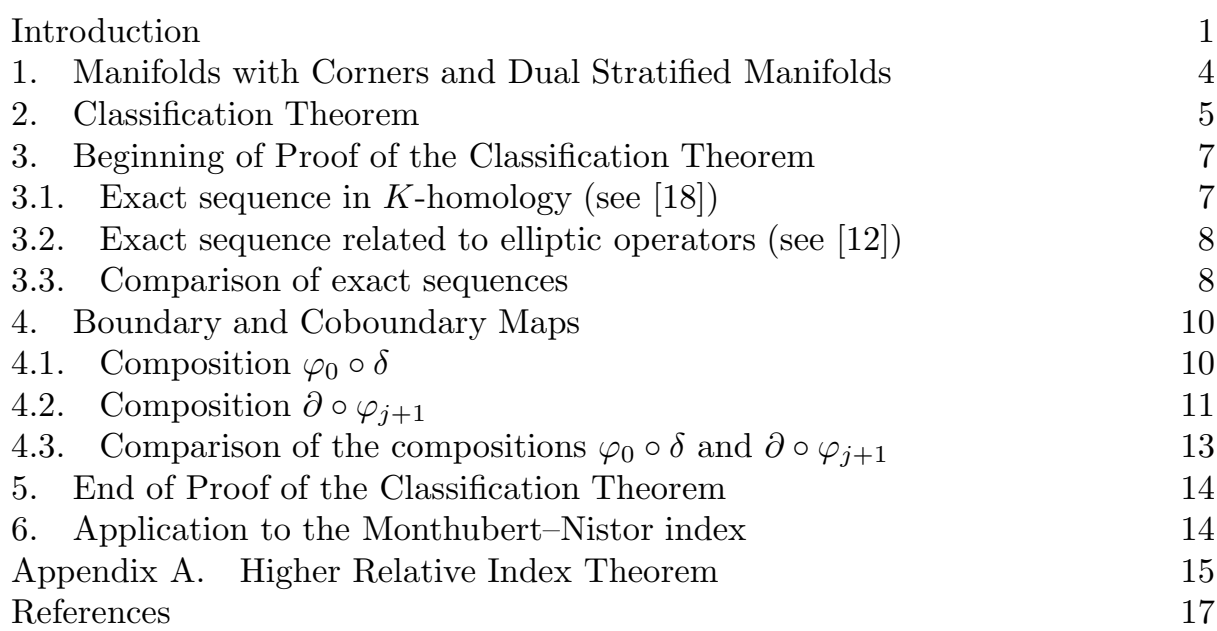

\section{INTRODUCTION}

Recently there has been considerable progress in understanding the notion of ellipticity on noncompact manifolds and manifolds with singularities. For a wide class of manifolds, ellipticity conditions for operators were established and the corresponding finiteness theorems ${ }^{1}$ were proved; the corresponding operator $C^{*}$-algebras were constructed. Hence the study of topological aspects of the theory of elliptic

1991 Mathematics Subject Classification. Primary 46L80; Secondary 19K33, 58J40.

Key words and phrases. Manifold with corners, elliptic operator, stable homotopy, Khomology, stratified manifold.

Supported in part by RFBR grants 05-01-00982 and 06-01-00098, by President of the Russian Federation grant MK-1713.2005.1, and by the DFG project 436 RUS 113/849/0-1® "K-theory and Noncommutative Geometry of Stratified Manifolds".

${ }^{1}$ Stating that an elliptic operator is Fredholm in certain function spaces. 
operators becomes topical. Here one mainly speaks of the classification problem and the index problem. Note that Gelfand's homotopy classification problem for elliptic operators can naturally be restated in modern language as the problem of computing the $K$-groups of symbol algebras, which prove noncommutative in most cases. Thus Gelfand's problem naturally fits in the framework of topical problems of Connes's noncommutative geometry [4].

Aim of the paper. This paper deals with elliptic theory on manifolds with corners.

Operators on manifolds with corners have been actively studied, and a number of important interesting results emerged recently. For example, the $C^{*}$-closure of symbol algebras was studied in [12], and a spectral sequence converging to the $K$ theory of the $C^{*}$-algebra of symbols was constructed. Monthubert 14 obtained a description of the operator algebra in the spirit of noncommutative geometry in terms of a special groupoid that can be associated with a manifold with corners (see also [9]). Bunke [3] constructed index theory of Atiyah-Patodi-Singer type for Dirac operators and studied cohomological obstructions to elliptic problems (see also [10, 8]); Monthubert and Nistor [15] produced a formula for the boundary map in the $K$-theory of symbol algebras in topological terms. Krainer [7] studied boundary value problems in this setting.

Although these results permitted finding the group classifying the homotopy classes of elliptic operators in a number of special cases (e.g., see [13 or [16]), the homotopy classification problem remained open.

We solve Gelfand's problem for manifolds with corners. Our goal is to obtain a simple explicit formula for the classifying group in terms of Atiyah's $K$-homology functor 1 .

Elliptic operators and $K$-homology. Note that the idea of classifying elliptic operators by the $K$-homology functor has long been known. For the reader's convenience, we recall it using operators on a smooth compact manifold $M$ as an example.

The commutator of an elliptic zero-order ${ }^{2}$ operator $D$ on $M$ with the operator of multiplication by a continuous function $f \in C(M)$ is compact

$$
[D, f] \in \mathcal{K} .
$$

By one definition, the contravariant $K$-theory $K^{0}(C(M))$ of the algebra $C(M)$ just consists of Fredholm operators for which the commutators (0.1) are compact. Thus $D$ determines an element of the group $K^{0}(C(M))$, which is isomorphic to the $K$-homology group of $M$ :

$$
K^{0}(C(M)) \simeq K_{0}(M)
$$

by the Atiyah-Brown-Douglas-Fillmore-Kasparov theorem. Thus, assigning the corresponding class in the $K$-homology to each elliptic operator, we obtain a mapping

$$
\operatorname{Ell}(M) \longrightarrow K_{0}(M)
$$

where $\operatorname{Ell}(M)$ is the group of elliptic operators in sections of bundles on $M$ modulo stable homotopy and $K_{0}(M)$ is the even $K$-homology group of $M$.

Kasparov showed this mapping to be an isomorphism. In other words, the $K$ homology group of a smooth manifold classifies elliptic operators on this manifold modulo stable homotopy.

\footnotetext{
${ }^{2}$ Working solely with zero-order operators does not result in loss of generality, since order reduction (say, multiplication by an appropriate power of the Laplace operator) is always available.
} 
This approach to classification also proved fruitful in the case of compact stratified manifolds with singularities. Namely, it was shown in 18 that in this case the even $K$-homology group of the underlying compact topological space classifies elliptic operators on this manifold.

However, no classification results were known for manifolds with corners of codimension $\geq 2$. The classification in the form of the $K$-homology of the manifold with corners, which suggests itself, is too meagre to be true: one can always smooth the corners, and we see that the $K$-homology of the manifold with corners is too coarse an invariant, for it does not take into account the structure of a manifold with corners.

Moreover even the space whose $K$-homology would classify elliptic operators was unknown.

Main result. We establish the isomorphism

$$
\operatorname{Ell}(M) \simeq K_{0}\left(M^{\#}\right),
$$

where $M$ is a manifold with corners and $M^{\#}$ is the dual manifold (see 19]) which is a stratified manifold with singularities. More precisely, the isomorphism (0.2) will be established under the following assumption concerning the combinatorial structure of the faces of our manifold:

\section{The normal bundles of all faces of $M$ are trivial.}

If this assumption fails, then, generally speaking, the isomorphism (0.2) does not hold. In this case, one should abandon the search for a classifying space and seek some algebra whose $K$-cohomology classifies elliptic operators. This algebra proves to be noncommutative, and one needs to use ideas on noncommutative geometry. These results will be considered elsewhere.

Note an interesting special case: if a manifold with corners is a polyhedron with a given triangulation of the boundary, then the dual stratified space is also a polyhedron, namely, the one used in the classical proof of Poincaré duality in cohomology! For example, if $M$ is a cube, then $M^{\#}$ is an octahedron. Thus the construction of the dual manifold in the first part [19] of the present paper generalizes the Poincaré dual polyhedron to the case of noncontractible faces.

Manifolds with corners and manifolds with multicylindrical ends. Note that there is a different perspective on the theory of operators on manifolds with corners. An application of a logarithmic change of variables in a neighborhood of the boundary taking the boundary to infinity (see Fig. 0.1 where this is shown for manifolds with boundary) results in the class of so-called manifolds with multicylindrical ends. These two pictures give the same operator algebras. Thus the results of the present paper also provide classification on manifolds with multicylindrical ends.

Outline of the paper. This is the second of the two parts of the paper. In the first part [19, the dual manifold of a manifold with corners was constructed and calculus of pseudodifferential operators $(\psi \mathrm{DO})$ on manifolds with corners was developed in the $C^{*}$-algebraic context.

The present part has the following structure.

In the first section, we recall some information from [19. In Sec. 2, we state the classification theorem. The proof occupies the next three sections. Note that the general scheme of the proof is the same as in 18, and we proceed by induction, passing from a smooth manifold to increasingly complex manifolds with 


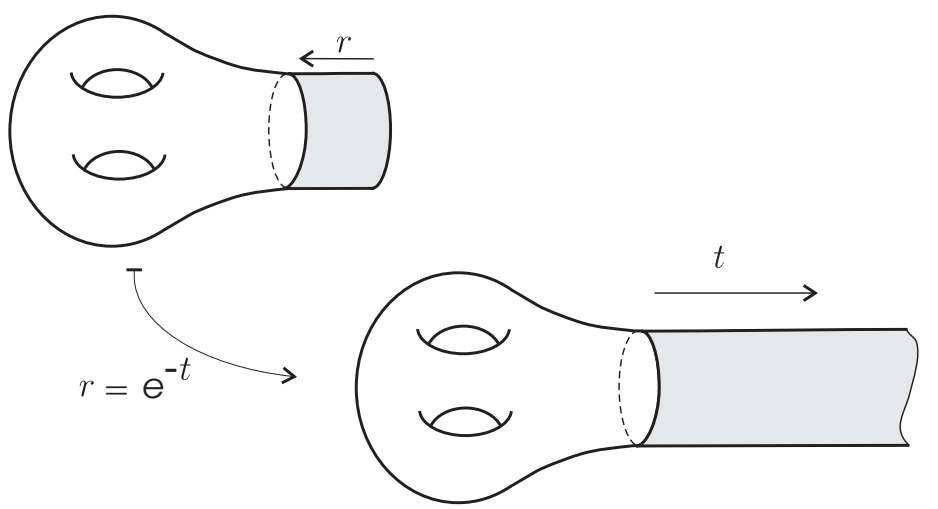

FiguRE 0.1. Transition from a neighborhood of the boundary to an infinite cylinder.

singularities. In Sec. 6 we discuss the relationship with some results due to Monthubert and Nistor. As a consequence of the classification theorem, we obtain a $K$-homology criterion for the vanishing of the index and a formula for the $K$-group of the $C^{*}$-algebra of $\psi \mathrm{DO}$ with zero interior symbol (this algebra corresponds to the $C^{*}$-algebra of the groupoid constructed by Monthubert). In the appendix, we prove a higher analog of the relative index theorem, which naturally arises when we obtain the classification of operators.

\section{Manifolds with Corners and Dual Stratified Manifolds}

Manifolds with corners and faces. Here we recall some information given in 19.

Consider a manifold $M$ with corners of depth $k$. It has a natural stratification

$$
M=\bigcup_{j=0}^{k} M_{j}
$$

where the stratum $M_{0}=M^{\circ}$ is just the interior of $M$ and each stratum $M_{j}$ is the union of connected components, open faces $M_{j \alpha}$ of codimension $j$ in $M$.

Each face $F=M_{j a}$ in the stratum $M_{j}$ is isomorphic to the interior of a manifold $\bar{F}=\bar{M}_{j a}$, which will be called a closed face of $M$. Faces of codimension one are called hyperfaces.

Main assumption. The main results of the paper will be obtained under the following assumption.

Assumption 1.1. The normal bundle $N_{+} F$ of each face $F$ is trivial.

In this case, the local defining functions $\rho_{1}, \ldots, \rho_{j}$ of $F$ are globally defined as functions on the normal bundle $N_{+} F$.

Remark. Assumption 1.1 holds if all hyperfaces are embedded, i.e., if there exists a global defining function for each hyperface $F \subset M$. However, it also holds, for some manifolds with nonembedded hyperfaces, say, for the raindrop. The simplest example of a manifold with corners that does not satisfy Assumption 1.1 is the Klein bottle with raindrop instead of the circle as the base. 
The dual space. The dual space $M^{\#}$ of a manifold $M$ with corners was introduced in [19]. If the original manifold is represented as the union

$$
M=\bigcup_{j \geq 0} M_{j}, \quad M_{j}=\bigcup_{\alpha} M_{j \alpha},
$$

then $M^{\#}$ is the union of dual faces,

$$
M^{\#}=\bigcup_{j \geq 0} M_{j}^{\#}, \quad M_{j}^{\#}=\bigcup_{\alpha} M_{j \alpha}^{\#},
$$

each of which is isomorphic to the interior of a simplex

$$
M_{j \alpha}^{\#} \simeq \Delta_{j-1}^{\circ} .
$$

Here, by definition, $M_{0}^{\#}:=M_{0}$ is the interior of $M$. Thus to each face $F$ of codimension $j$ in $M$ there corresponds a simplex $F^{\#}$ of dimension $j-1$ in the dual space.

The fibration structure on $M^{\#}$. It was proved in 19 that a neighborhood $U^{\#}$ of the stratum $F^{\#}$ is homeomorphic to the product of $F^{\#}$ by the cone

$$
K_{\Omega}=[0,1) \times \Omega /\{0\} \times \Omega
$$

whose base $\Omega$ is the dual space $\bar{F}^{\#}$ of the closed face $\bar{F}$ (which is well defined, since $\bar{F}$ itself is a manifold with corners). As a result, we find that $M^{\#}$ is a stratified manifold with singularities.

\section{Classification Theorem}

Let $M$ be a manifold with corners satisfying Assumption 1.1 and let $\Psi(M)$ be the $C^{*}$-algebra of zero-order $\psi \mathrm{DO}$ in the space $L^{2}(M)$ (see 19]). The notion of a $\psi \mathrm{DO}$ acting on sections of finite-dimensional vector bundles on $M$ is introduced in the usual way. There is a natural equivalence relation, stable homotopy, on the set of elliptic operators. Recall the definition.

Definition 2.1. Two elliptic operators

$$
D: L^{2}(M, E) \rightarrow L^{2}(M, F) \text { and } D^{\prime}: L^{2}\left(M, E^{\prime}\right) \rightarrow L^{2}\left(M, F^{\prime}\right)
$$

on $M$ are said to be stably homotopic if there exists a continuous homotopy

$$
D \oplus 1_{E_{0}} \sim f^{*}\left(D^{\prime} \oplus 1_{F_{0}}\right) e^{*}
$$

of elliptic operators, where $E_{0}, F_{0} \in \operatorname{Vect}(M)$ are vector bundles and

$$
e: E \oplus E_{0} \longrightarrow E^{\prime} \oplus F_{0}, \quad f: F^{\prime} \oplus F_{0} \longrightarrow F \oplus E_{0}
$$

are bundle isomorphisms.

Here ellipticity is understood as the invertibility of all components of the symbol of the operator, and only homotopies of $\psi \mathrm{DO}$ preserving ellipticity are considered.

Even groups $\operatorname{Ell}_{0}(M)$. Stable homotopy is an equivalence relation on the set of elliptic $\psi \mathrm{DO}$ acting in sections of vector bundles. By $\operatorname{Ell}_{0}(M)$ we denote the corresponding quotient set. It is a group with respect to the direct sum of operators, and the inverse in this group is given by the coset of the almost inverse operator (i.e., an inverse modulo compact operators). 
Odd groups $\operatorname{Ell}_{1}(M)$. Odd elliptic theory $\operatorname{Ell}_{1}(M)$ is defined in a similar way as the group of stable homotopy classes of elliptic self-adjoint operators. Stabilization is defined in terms of the operators $\pm I d$.

Remark 2.2. An equivalent definition of the odd Ell-group can be given in terms of smooth operator families on $M$ with parameter space $\mathbb{S}^{1}$ modulo constant families.

We shall compute the groups $\operatorname{El}_{*}(M)$ for $*=0$ and $*=1$, i.e., find the classification of elliptic operators modulo stable homotopy. Our approach is based on the following fact (see the definition of $\psi \mathrm{DO}$ in [19]):

$\psi D O$ on $M$ can be viewed as local operators in the sense of Atiyah on the dual manifold $M^{\#}$.

Thus an elliptic $\psi$ DO defines a Fredholm module on the space $L^{2}(M)$ viewed as a $C\left(M^{\#}\right)$-module. (For Fredholm modules and $K$-theory, see [5] or [2]).

Classification of elliptic operators. The following theorem is the main result of this paper.

Theorem 2.3. The mapping that takes each elliptic $\psi D O$ to the corresponding Fredholm module defines the group isomorphism

$$
\operatorname{Ell}_{*}(M) \stackrel{\simeq}{\longrightarrow} K_{*}\left(M^{\#}\right) .
$$

We shall obtain this theorem as a special case of the following more general theorem.

Classification of partially elliptic operators (cf. [18]). Let $\operatorname{Ell}_{*}\left(M_{\geq j}\right)$ be the group generated by operators whose symbols are invertible on the main stratum and all faces of codimension $\geq j$. Thus we consider operators satisfying the ellipticity condition on part of the faces.

The corresponding dual space

$$
M_{\geq j}^{\#}:=M^{\#} \backslash \bigcup_{j^{\prime}=1}^{j-1} M_{j^{\prime}}^{\#}
$$

is obtained from $M^{\#}$ by deleting all simplices of dimension $\leq j-2$.

Lemma 2.4. An operator $D$ such that $[D] \in \operatorname{Ell}_{*}\left(M_{\geq j}\right)$ defines a Fredholm module over the algebra $C_{0}\left(M_{\geq j}^{\#}\right)$ of functions on the dual space.

Proof. We should verify the following properties of a Fredholm module: the expression

$$
f\left(D D^{*}-1\right)
$$

is compact for all $f \in C_{0}\left(M_{\geq j}^{\#}\right)$ (here we assume that $D$ is normalized by the condition $\sigma_{s}^{*}(D)=\sigma_{s}(D)^{-1}$ for $s \geq j$ ). The compactness follows from the fact that, by construction, on each face $F \subset M$ either the corresponding symbol of our operator is invertible or the functions in the algebra $C_{0}\left(M_{\geq j}^{\#}\right)$ are zero.

By Lemma 2.4 the mapping

$$
\mathrm{Ell}_{*}\left(M_{\geq j}\right) \stackrel{\varphi_{j}}{\longrightarrow} K_{*}\left(M_{\geq j}^{\#}\right)
$$

that takes partially elliptic operators to the corresponding Fredholm modules is well defined (cf. [18]). 
Theorem 2.5. For each $1 \leq j \leq k+1$, the mapping (2.2) is an isomorphism.

Theorem 2.3 is the special case of Theorem 2.5 for $j=1$.

\section{Beginning of Proof of the Classifichtion Theorem}

We prove Theorem 2.5 by induction on $j$ decreasing from $k+1$ (where $k$ is the depth of $M)$ to 1.

Inductive assumption. For $j=k+1$, the group $\operatorname{Ell}_{*}\left(M_{\geq j}\right)$ classifies elliptic interior symbols and hence is isomorphic to $K_{c}^{*}\left(T^{*} M\right)$. Moreover, the mapping taking the symbol to the corresponding operator determines an isomorphism $K_{c}^{*}\left(T^{*} M\right) \simeq$ $K_{*}\left(M_{0}\right)$ (e.g., see [6). On the other hand, the right-hand side of (2.2) in this case just contains the group $K_{*}\left(M_{0}\right)$. Thus the theorem holds for $j=k+1$.

Inductive step. To justify the inductive step, we need to study exact sequences in $K$-homology and $K$-theory permitting one to relate the maps $\varphi_{j}$ in (2.2) for two values of the subscript, $j$ and $j+1$.

\subsection{Exact sequence in $K$-homology (see [18]). Consider the embedding}

$$
M_{\geq j}^{\#} \supset M_{j}^{\#} .
$$

The complement $M_{\geq j}^{\#} \backslash M_{j}^{\#}$ is obviously equal to $M_{\geq j+1}^{\#}$, and we have the exact sequence of the pair (3.1) in $K$-homology

$$
\ldots \rightarrow K_{*}\left(M_{j}^{\#}\right) \rightarrow K_{*}\left(M_{\geq j}^{\#}\right) \rightarrow K_{*}\left(M_{\geq j+1}^{\#}\right) \stackrel{\partial}{\rightarrow} K_{*+1}\left(M_{j}^{\#}\right) \rightarrow \ldots
$$

All maps but the boundary map $\partial$ in this sequence correspond to a change of module structure on the corresponding Fredholm modules. The boundary map $\partial$ can be reduced to a form convenient for computations by the following standard method.

Let $U^{\#} \subset M_{>j}^{\#}$ be the open neighborhood of the stratum $M_{j}^{\#}$ constructed $^{3}$ in 19. Sec. 1.2]. We have the homeomorphism

$$
U^{\#} \simeq M_{j}^{\#} \times K_{\bar{M}_{j}}^{\#},
$$

where the cone $K_{\Omega_{j}}$ is the disjoint union of the cones corresponding to the connected components of the base $\Omega_{j}$.

Then we have the mappings

$$
M_{j}^{\#} \times(0,1) \stackrel{\pi}{\longleftarrow} M_{j}^{\#} \times(0,1) \times \Omega_{j} \stackrel{\widetilde{\leftarrow}}{\leftarrow} U^{\#} \backslash M_{j}^{\#} \stackrel{l}{\longrightarrow} M_{\geq j+1}^{\#}
$$

(by $l$ we denote the embedding of an open manifold, and $\pi$ is the projection onto the first two factors), which permit us to represent the boundary map $\partial$ in (3.2) as the composition

$$
K_{*}\left(M_{\geq j+1}^{\#}\right) \stackrel{l^{*}}{\rightarrow} K_{*}\left(U^{\#} \backslash M_{j}^{\#}\right) \stackrel{\pi_{*}}{\rightarrow} K_{*}\left((0,1) \times M_{j}^{\#}\right) \stackrel{\beta}{\simeq} K_{*+1}\left(M_{j}^{\#}\right)
$$

of the restriction $l^{*}$ of operators to an open set, the push-forward $\pi_{*}$, and the periodicity isomorphism $\beta$. This representation follows from the fact that $\partial$ is natural.

\footnotetext{
${ }^{3}$ The neighborhood $U$ is defined as the union of neighborhoods of all simplices $F^{\#} \subset M_{j}^{\#}$. By construction, these neighborhoods are disjoint.
} 
3.2. Exact sequence related to elliptic operators (see [12]). Let $M$ be a manifold with corners of depth $k>0$, and let $j, 1 \leq j \leq k$, be some number. We denote the algebra formed by the symbols $\left(\sigma_{0}, \sigma_{j}, \sigma_{j+1}, \ldots, \sigma_{k}\right)$ of all $\psi \mathrm{DO}$ on $M$ by

$$
\Sigma_{j}=\operatorname{Im}\left(\sigma_{0}, \sigma_{j}, \sigma_{j+1}, \ldots, \sigma_{k}\right) .
$$

Then we have the short exact sequence of $C^{*}$-algebras

$$
0 \rightarrow J \rightarrow \Sigma_{j} \rightarrow \Sigma_{j+1} \rightarrow 0 .
$$

Here the ideal $J$ consists of the symbols $\left(\sigma_{0}, \sigma_{j}, \sigma_{j+1}, \ldots, \sigma_{k}\right)$ in which all components but $\sigma_{j}$ are zero. From the compactness criterion for $\psi \mathrm{DO}$ and compatibility conditions for symbols (see [19]), we see that under these conditions the symbol $\sigma_{j}$ is a tuple of compact-valued families decaying at infinity, so that one has the isomorphism

$$
J \simeq \bigoplus_{F \subset M_{j}} C_{0}\left(\mathbb{R}^{j}, \mathcal{K} L^{2}(F)\right)
$$

where the sum is taken over faces $F$ of codimension $j$ in $M$.

By virtue of this isomorphism, we can write out the exact sequence in $K$-theory corresponding to the short sequence (3.5) in the form

$$
\ldots \rightarrow K_{*}(J) \rightarrow K_{*}\left(\Sigma_{j}\right) \rightarrow K_{*}\left(\Sigma_{j+1}\right) \stackrel{\delta}{\longrightarrow} K_{*+1}(J) \rightarrow \ldots
$$

Clearly,

$$
K_{*}(J) \simeq K_{*}\left(C_{0}\left(\mathbb{R}^{j}\right)\right) \oplus K_{*}\left(C_{0}\left(\mathbb{R}^{j}\right)\right) \oplus \ldots=\mathbb{Z}^{l},
$$

where $l$ is the number of connected components in $M_{j}$. In terms of this isomorphism, the boundary map $\delta$ can be represented (for $*=1$ ) in the following simple form. An arbitrary class

$$
[\sigma] \in K_{1}\left(\Sigma_{j+1}\right)
$$

is realized by an invertible symbol

$$
\sigma=\left(\sigma_{0}, \sigma_{j+1}, \sigma_{j+2}, \ldots, \sigma_{k}\right) .
$$

(From now on, for brevity we carry out the computations only for $K$-theory elements representable by scalar operators; the consideration of the matrix case differs only in the awkwardness of formulas.) Take an arbitrary symbol $\sigma_{j}$ compatible with $\sigma$. The symbol $\sigma_{j}$ defines an elliptic family with parameters in $\mathbb{R}^{j}$, and the index of that family is a well-defined element of the $K$-group with compact supports of the parameter space. One has

$$
\delta[\sigma]=\operatorname{ind} \sigma_{j} \in \bigoplus_{F \subset M_{j}} K_{0}\left(C_{0}\left(\mathbb{R}^{j}\right)\right) .
$$

There is a similar expression for the boundary map for the case $*=0$. (To obtain it, one can pass to the suspension.)

3.3. Comparison of exact sequences. Let us show that the sequences (3.2) and (3.6) can be combined into the commutative diagram

$$
\begin{aligned}
& \ldots \rightarrow K_{*}(J) \quad \rightarrow K_{*}\left(\Sigma_{j}\right) \rightarrow \quad K_{*}\left(\Sigma_{j+1}\right) \quad \stackrel{\delta}{\longrightarrow} K_{*+1}(J) \quad \rightarrow \ldots \\
& \downarrow \varphi_{0} \quad \downarrow \varphi_{j} \quad \downarrow \varphi_{j+1} \quad \downarrow \varphi_{0} \\
& \ldots \rightarrow K_{*+1}\left(M_{j}^{\#}\right) \rightarrow K_{*+1}\left(M_{\geq j}^{\#}\right) \rightarrow K_{*+1}\left(M_{\geq j+1}^{\#}\right) \stackrel{\partial}{\longrightarrow} K_{*}\left(M_{j}^{\#}\right) \rightarrow \ldots
\end{aligned}
$$


(The construction of this diagram and the verification of its commutativity will be finished in Sec. 4.3]

First, we define the vertical maps in the diagram. Without loss of generality, we can assume that $M$ has no connected components with empty boundary. (Everybody knows the classification on such components.) Then for all $j$ we have the isomorphism $[17]^{4}$

$$
K_{*}\left(\Sigma_{j}\right) \simeq \operatorname{Ell}_{*+1}\left(M_{\geq j}\right) \oplus K_{*}(C(M)) .
$$

Hence we define the maps $\varphi_{j}, j \geq 1$, in diagram (3.7) as the composition

$$
K_{*}\left(\Sigma_{j}\right) \longrightarrow \operatorname{Ell}_{*+1}\left(M_{\geq j}\right) \longrightarrow K_{*+1}\left(M_{\geq j}^{\#}\right)
$$

of the projection onto the Ell-group and the quantization (2.2). Thus these maps are induced by quantization, which takes symbols to operators.

It remains to define the map $\varphi_{0}$. Just as the other vertical arrows in the diagram, it is defined by quantization, namely, by quantization of symbols $\sigma=\sigma_{j}$ in the ideal $J$. The quantization of elements of the ideal differs from the quantization of general elements of the algebra $\Sigma_{j}$ only in that the operator is considered in the $L^{2}$ space in a small neighborhood $U$ of the stratum $M_{j}$ in $M$ constructed in [19, Lemma 1.9]. We denote the operator by $\widehat{\sigma}_{j}$.

Let us define a module structure on $L^{2}(U)$. To this end recall that $U$ can be considered also as a subset of the positive quadrant $N_{+}^{\prime} M_{j}$ of the logarithmic normal bundle. Thus, this space is naturally a $C_{0}\left(M_{j}^{\#}\right)$-module. (Elements of $C_{0}\left(M_{j}^{\#}\right)$ act on $N_{+}^{\prime} M_{j}$ as operators of multiplication by radially constant functions $f(y)$, in $\operatorname{logarithmic}$ coordinates $y=-\ln \rho$.) The verification of locality of operator with respect to this module structure (i.e., proving that the operator $\widehat{\sigma}_{j}$ commutes with operators of multiplication by functions modulo compact operators) is immediate, and hence for the element $\left[\sigma_{j}\right] \in K_{*+1}(J)$ we define the element

$$
\varphi_{0}\left[\sigma_{j}\right]:=\left[\widehat{\sigma}_{j}\right] \in K_{*}\left(M_{j}^{\#}\right) \text {. }
$$

Diagram (3.7) commutes. The commutativity of the middle square of the diagram follows directly from definitions.

Lemma 3.1. The left square of diagram (3.7) commutes.

Proof. Indeed, consider the composition of mappings passing through the right upper corner of the square

$$
\begin{array}{rcc}
K_{*}(J) & \rightarrow & K_{*}\left(\Sigma_{j}\right) \\
\downarrow \varphi_{0} & & \downarrow \varphi_{j} \\
K_{*+1}\left(M_{j}^{\#}\right) & \rightarrow & K_{*+1}\left(M_{\geq j}^{\#}\right) .
\end{array}
$$

It takes an elliptic symbol $\sigma_{j}$ to the operator $\widehat{\sigma}_{j}$, which acts outside a neighborhood $U$ of the stratum $M_{j}^{\#}$ as the identity operator (modulo compact operators) in the space $L^{2}(M)$ with the natural structure of a $C_{0}\left(M_{\geq j}^{\#}\right)$-module. Now if we restrict the operator to a neighborhood of $M_{j}$ (the element in $K$-homology remains

\footnotetext{
${ }^{4}$ This isomorphism generalizes the well-known expansion $K^{1}\left(S^{*} M\right) \simeq \operatorname{Ell}(M) \oplus K^{1}(M)$ on a smooth closed manifold $M$ on which there exists a nonzero vector field. Elimination of closed components permits us to claim that there exists a nonzero vector field in our situation.
} 
unchanged, since the corresponding Fredholm module changes by a degenerate module) and then use a homotopy to reduce the module structure to the composition

$$
C_{0}\left(M_{\geq j}^{\#}\right) \stackrel{i^{*}}{\rightarrow} C_{0}\left(M_{j}^{\#}\right) \stackrel{\pi^{*}}{\rightarrow} C\left(U^{\#}\right) \rightarrow \mathcal{B}\left(L^{2}(U)\right),
$$

where $\pi: U^{\#} \rightarrow M_{j}^{\#}$ is a projection and $i: M_{j}^{\#} \subset M_{\geq j}^{\#}$ is an embedding, then we obtain the Fredholm module assigned to the symbol $\sigma_{j}$ by the composition of maps passing through left bottom corner of the square. The commutativity of the square is thereby established.

Verification of the commutativity of the square containing the boundary maps is rather cumbersome, and so we make it in a separate section.

\section{BOUndary AND COBOUNDARY MAPS}

In this section, we establish the commutativity of the square

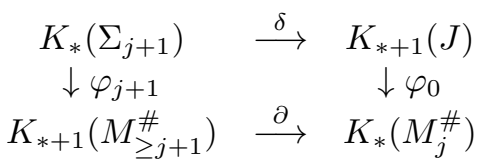

containing the boundary maps in diagram (3.7). The scheme of proof is as follows. We

(1) Compute the composition $\varphi_{0} \circ \delta$.

(2) Compute the composition $\partial \circ \varphi_{j+1}$.

(3) Compare the resulting expressions.

4.1. Composition $\varphi_{0} \circ \delta$. Let $[\sigma] \in K_{*}\left(\Sigma_{j+1}\right)$ be the element defined by some symbol $\sigma=\left(\sigma_{0}, \sigma_{j+1}, \ldots, \sigma_{k}\right)$. Take a symbol $\sigma_{j}$ on $M_{j}$ compatible with $\sigma$ and denote by

$$
\widehat{\sigma}_{j}: L^{2}\left(N M_{j}\right) \rightarrow L^{2}\left(N M_{j}\right)
$$

the corresponding translation-invariant infinitesimal operator. (It is conjugate to $\sigma_{j}$ by the Fourier transform.)

Representing the space $N_{+}^{\prime} M_{j}$ as the product $N_{+}^{\prime} M_{j} \simeq M_{j} \times \mathbb{R}_{+}^{j}$, we see that $L^{2}\left(N M_{j}\right)$ is a $C_{0}\left(\bigsqcup \mathbb{R}_{+}^{j}\right)$-module. Here $\bigsqcup \mathbb{R}_{+}^{j}$ is the disjoint union of as many open quadrants as there are faces of codimension $j$ in $M$. The operator $\widehat{\sigma}_{j}$ is local with respect to this module structure. We denote the corresponding element of the $K$-homology group by

$$
\left[\widehat{\sigma}_{j}\right] \in K_{*+1}\left(\bigsqcup \mathbb{R}_{+}^{j}\right) .
$$

Lemma 4.1. The element $[\sigma] \in K_{*}\left(\Sigma_{j+1}\right)$ satisfies the chain of relations

$$
\varphi_{0} \delta[\sigma]=\varphi_{0}\left(\operatorname{ind} \sigma_{j}\right)=\beta\left[\widehat{\sigma}_{j}\right] \in K_{*}\left(M_{j}^{\#}\right),
$$

where $\beta: K_{*+1}\left(\bigsqcup \mathbb{R}_{+}^{j}\right)=K_{*+1}\left(M_{j}^{\#} \times \mathbb{R}_{+}\right) \longrightarrow K_{*}\left(M_{j}^{\#}\right)$ is the Bott periodicity isomorphism, and the index is understood as the index

$$
\text { ind } \sigma_{j} \in K^{*+1}\left(\bigsqcup \mathbb{R}^{j}\right) \simeq K_{*+1}(J)
$$

of the elliptic operator-valued symbol $\sigma_{j}$. 
Proof. For brevity, we assume that $M_{j}$ consists of exactly one stratum. In this case, we have $M_{j}^{\#} \times \mathbb{R}_{+} \simeq \mathbb{R}_{+}^{j}$.

The first relation in (4.4) follows from definitions (since the boundary map in $K$-theory of algebras is the index map).

1. Let us establish the second relation $\varphi_{0}\left(\right.$ ind $\left.\sigma_{j}\right)=\beta\left[\widehat{\sigma}_{j}\right]$. The proof is based on the diagram

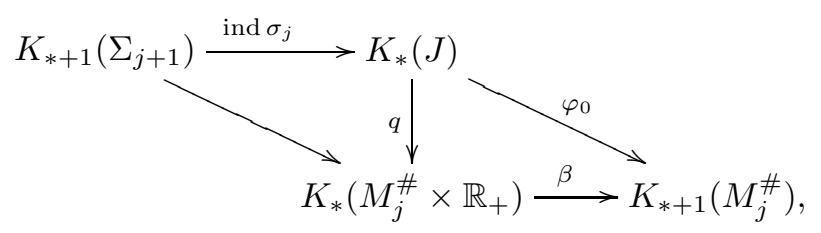

where the map $K_{*+1}\left(\Sigma_{j+1}\right) \rightarrow K_{*}\left(M_{j}^{\#} \times \mathbb{R}_{+}\right)$is induced by the map that takes the symbol $\sigma$ to the operator $\widehat{\sigma}_{j}$ in (4.3). Finally, the group $K_{*}(J) \simeq K^{*}\left(\mathbb{R}^{j}\right)$ is interpreted as the $K$-group of the cotangent bundle to $\mathbb{R}_{+}^{j}$, and the map $q$ is induced by standard quantization (to a symbol on the cosphere bundle, one assigns a pseudodifferential operator).

2. We claim that diagram (4.5) commutes. Indeed, let us verify the commutativity of the left triangle, i.e., the relation

$$
\left[\widehat{\sigma}_{j}\right]=q\left[\text { ind } \sigma_{j}\right] .
$$

Note that the operator $\widehat{\sigma}_{j}$ is given over the product $N M_{j}=M_{j} \times \mathbb{R}^{j}$. Moreover, it can be viewed as a $\psi \mathrm{DO}$ on $\mathbb{R}^{j}$ with operator-valued symbol $\sigma_{j}=\sigma_{j}(\xi), \xi \in \mathbb{R}^{j}$. This symbol is independent of the physical variables $x \in \mathbb{R}^{j}$. Without loss of generality, it can be assumed to be smooth with respect to the parameter $\xi$ (since $\sigma_{j}(\xi)$, just as any $\psi \mathrm{DO}$ with a parameter, can be arbitrarily closely approximated by a smooth $\psi \mathrm{DO}$ with a parameter; see [19]). Hence $\sigma_{j}(\xi)$ is an operator-valued symbol in the sense of [11, i.e., has a compact variation with respect to $\xi$ and all of its derivatives starting from the first decay at infinity. Now relation (4.6) follows by analogy with the generalized Luke theorem in [18.

The commutativity of the right triangle follows (see Corollary A.2 in the appendix) from the higher relative index theorem.

3. The commutativity of diagram (4.5) implies the second relation (4.4). (The right-hand side is obtained if from the left top corner of the diagram we go directly to the group $K_{*}\left(M_{j}^{\#} \times \mathbb{R}_{+}\right)$and then apply the periodicity isomorphism $\beta$.)

\subsection{Composition $\partial \circ \varphi_{j+1}$.}

Space $N_{+}^{\prime} M_{j}$ as a manifold with corners. The image of the positive quadrant $N_{+}^{\prime} M_{j}$ under the inverse of the logarithmic map is the set $M_{j} \times[0,1)^{j} \subset M_{j} \times \overline{\mathbb{R}}_{+}^{j}=$ $N_{+} M_{j}$. Hence we treat $N_{+}^{\prime} M_{j}$ as the interior of a manifold with corners, denoted by $\overline{N_{+}^{\prime} M_{j}}$. We denote the corresponding dual space by ${\overline{N_{+}^{\prime} M_{j}}}^{\#}$. On the complement ${\overline{N_{+}^{\prime}}}_{M_{j}}^{\#} \backslash M_{j}^{\#}$, there is a well-defined projection

$$
\begin{array}{clc}
{\overline{N_{+}^{\prime} M_{j}}}^{\#} \backslash M_{j}^{\#} & \stackrel{\pi}{\longrightarrow} & M_{j}^{\#} \times \mathbb{R}_{+} \\
(y, x, \omega) & \mapsto & \left(\frac{y}{|y|}, \frac{|x|+1}{|y|}\right),
\end{array}
$$


whose fiber is the space $\bar{M}_{j}{ }^{\#}$.

Reduction into a neighborhood of the edge. We have the diagram of embeddings

$$
\begin{aligned}
& M_{j}^{\#} \subset \quad U^{\#} \quad \subset M_{\geq j}^{\#} \\
& \frac{\bigcap}{N_{+}^{\prime} M_{j}}{ }^{\#} \text {. }
\end{aligned}
$$

Let $[\sigma] \in K_{*+1}\left(\Sigma_{j+1}\right)$ be the element determined by the symbol $\sigma$ as above. By passing to the corresponding operators, we obtain the element

$$
\varphi_{j+1}[\sigma] \in K_{*}\left(M_{\geq j+1}^{\#}\right) .
$$

On the other hand, the infinitesimal operator $\widehat{\sigma}_{j}$ compatible with $\sigma$ (see (4.2)) defines the element

$$
\left[\widehat{\sigma}_{j}\right]^{\prime} \in K_{*}\left({\overline{N_{+} M_{j}}}^{\#} \backslash M_{j}^{\#}\right) .
$$

This element is well defined, since the components of its symbol are elliptic on the corresponding strata. We use primes to distinguish this element from the element (4.3): although they are determined by one and the same operator, the module structures on the $L^{2}$-spaces are different.

The naturality of the boundary map in $K$-homology results in the following lemma.

Lemma 4.2. One has

$$
\partial \varphi_{j+1}[\sigma]=\partial^{\prime}\left[\widehat{\sigma}_{j}\right]^{\prime}
$$

where $\partial^{\prime}: K_{*}\left({\overline{N_{+}}}_{j}^{\#} \backslash M_{j}^{\#}\right) \rightarrow K_{*+1}\left(M_{j}^{\#}\right)$ is the boundary map for the pair $M_{j}^{\#} \subset{\overline{N_{+}}}_{j}{ }^{\#}$.

Proof. Let $D$ be some operator on $M$ with symbol $\sigma$.

1. The infinitesimal operator $\widehat{\sigma}_{j}$ is obtained from $D$ by localization to the set $M_{j}^{\#}$. Hence the restrictions $\left.D\right|_{U}$ and $\left.\widehat{\sigma}_{j}\right|_{U}$ of these operators to a small space $U$ of $M_{j}$ are connected by a linear homotopy; i.e., one has

$$
\left[\left.D\right|_{U}\right]=\left[\left.\widehat{\sigma}_{j}\right|_{U}\right] \in K_{*}\left(U^{\#}\right) .
$$

2. By applying the naturality of the boundary map in $K$-homology to the embedding diagram (4.8), we obtain

$$
\partial \varphi_{j+1}[\sigma] \equiv \partial[D]=\partial^{\prime \prime}\left[\left.D\right|_{U}\right]
$$

where $\partial^{\prime \prime}$ is the boundary map for the pair $M_{j}^{\#} \subset U^{\#}$. Now if on the right-hand side of the last relation we replace the element $\left[\left.D\right|_{U}\right]$ according to (4.9) and once more use the naturality of the boundary map, then we obtain the desired relation

$$
\partial \varphi_{j+1}[\sigma]=\partial^{\prime \prime}\left[\left.\widehat{\sigma}_{j}\right|_{U}\right]=\partial^{\prime}\left[\widehat{\sigma}_{j}\right]^{\prime}
$$

Thus in what follows, when computing the composition $\partial \circ \varphi_{j+1}$, we can (and will) work with the operator $\widehat{\sigma}_{j}$ on $N_{+}^{\prime} M_{j}$. 
Homotopy of the module structure. By (3.4), the boundary map $\partial^{\prime}$ in Lemma 4.2 can be represented as the composition

$$
K_{*}\left({\overline{N_{+}^{\prime}}}_{j}^{\#} \backslash M_{j}^{\#}\right) \stackrel{\pi_{*}}{\rightarrow} K_{*}\left(M_{j}^{\#} \times \mathbb{R}_{+}\right) \stackrel{\beta}{\rightarrow} K_{*+1}\left(M_{j}^{\#}\right)
$$

of the push-forward with respect to the projection $\pi$ and the periodicity isomorphism.

Unfortunately, although the classes $\left[\widehat{\sigma}_{j}\right]$ and $\pi_{*}\left[\widehat{\sigma}_{j}\right]^{\prime}$ are determined by the same operator $\widehat{\sigma}_{j}$, they have different module structures on the space $L^{2}\left(N M_{j}\right)$ : in the first case, the structure is independent of the coordinate $x$, while in the second case it depends on (see (4.10).

Let us make a homotopy of module structures. To this end, we define a homotopy

$$
\pi^{\varepsilon}:{\overline{N_{+}^{\prime}}}_{j}^{\#} \backslash M_{j}^{\#} \longrightarrow M_{j}^{\#} \times \mathbb{R}_{+}
$$

of projections by the formula (cf. (4.7))

$$
\pi^{\varepsilon}(y, x, \omega):=\left(\frac{y}{|y|}, \frac{\varepsilon|x|+1}{|y|}\right) .
$$

This formula defines a continuous family of maps for $\varepsilon>0$. However, the family is not continuous as $\varepsilon \rightarrow 0 .{ }^{5}$ Nevertheless, continuity takes place for the Fredholm modules, as shown by the following lemma.

Lemma 4.3. The family $\pi_{*}^{\varepsilon}\left(\widehat{\sigma}_{j}\right)^{\prime}$ of Fredholm modules obtained by the change of module structure defines a homotopy in the sense of $K K$-theory, and one has

$$
\lim _{\varepsilon \rightarrow 0} \pi_{*}^{\varepsilon}\left(\widehat{\sigma}_{j}\right)^{\prime}=\pi_{*}^{0}\left(\widehat{\sigma}_{j}\right)^{\prime},
$$

whence it follows that $\pi_{*}\left[\widehat{\sigma}_{j}\right]^{\prime}=\left[\pi_{*}^{0}\left(\widehat{\sigma}_{j}\right)\right]^{\prime} \in K_{*}\left(M_{j}^{\#} \times \mathbb{R}_{+}\right)$.

Proof. For brevity, we assume that $M_{j}$ consists of a single face, i.e., is connected. Then the homotopy in the sense of $K K$-theory means (e.g., see [2]) that for each function $f \in C_{0}\left(\mathbb{R}_{+}^{j}\right)$ the family

$$
g^{\varepsilon}=\left(\pi^{\varepsilon}\right)^{*}(f): L^{2}\left(N_{+}^{\prime} M_{j}\right) \longrightarrow L^{2}\left(N_{+}^{\prime} M_{j}\right)
$$

of operators of multiplication by the functions $\left(\pi^{\varepsilon}\right)^{*}(f)$ is strongly $*$-continuous and that the operator families

$$
\left[g^{\varepsilon}, \widehat{\sigma}_{j}\right], \quad g^{\varepsilon}\left(\widehat{\sigma}_{j} \widehat{\sigma}_{j}^{-1}-1\right)
$$

in $L^{2}\left(N M_{j}\right)$ are continuous families of compact operators as $\varepsilon \rightarrow 0$.

It suffices to prove all these facts for (a dense set of) smooth functions $f$. If $f$ is smooth, then one should smooth the family $g^{\varepsilon}$ and use the composition formulas, which provide the desired compactness and continuity.

4.3. Comparison of the compositions $\varphi_{0} \circ \delta$ and $\partial \circ \varphi_{j+1}$. Now let us use Lemmas 4.14.3. We obtain the chain of relations

$$
\begin{aligned}
& \partial \varphi_{j+1}[\sigma] \stackrel{\text { Lemma } 4.2}{=} \partial^{\prime}\left[\widehat{\sigma}_{j}\right]^{\text {formula }} \stackrel{4.10]}{=} \beta \pi_{*}^{1}\left[\widehat{\sigma}_{j}\right]^{\prime} \stackrel{\text { Lemma }}{=} \beta\left[\pi_{*}^{0} \widehat{\sigma}_{j}\right]^{\prime}=
\end{aligned}
$$

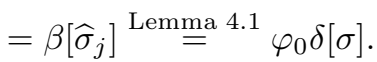

The equality at the end of the first row corresponds to the identical coincidence of the corresponding Fredholm modules.

\footnotetext{
${ }^{5}$ And hence the map $\pi_{*}^{0}$ is not defined on the $K$-group.
} 
Thus the square (4.1) commutes, and we have established the commutativity of diagram (3.7).

\section{End of Proof of the Classification Theorem}

By virtue of the isomorphism (3.8), we can single out and cancel the summand $K_{*}(C(M))$ in diagram (3.7) in the terms $K_{*}\left(\Sigma_{j}\right)$ and $K_{*}\left(\Sigma_{j+1}\right)$. We obtain the diagram

$$
\begin{array}{rccccc}
\ldots \rightarrow K_{*}(J) & \rightarrow \operatorname{Ell}_{*+1}\left(M_{\geq j}\right) \rightarrow & \operatorname{Ell}_{*+1}\left(M_{\geq j+1}\right) & \stackrel{\delta}{\rightarrow} & K_{*+1}(J) & \rightarrow \ldots \\
\downarrow \varphi_{0} & \downarrow \varphi_{j} & \downarrow \varphi_{j+1} & & \downarrow \varphi_{0} & \\
\ldots \rightarrow K_{*+1}\left(M_{j}^{\#}\right) & \rightarrow K_{*+1}\left(M_{\geq j}^{\#}\right) \rightarrow & K_{*+1}\left(M_{\geq j+1}^{\#}\right) & \stackrel{\partial}{\rightarrow} & K_{*}\left(M_{j}^{\#}\right) & \rightarrow \ldots
\end{array}
$$

The map $\varphi_{j+1}$ is an isomorphism by the inductive assumption. The map $\varphi_{0}$ is also an isomorphism (see Corollary A.2 in the Appendix). Since the diagram commutes, we can apply the 5-lemma and obtain the desired justification of the induction step in Theorem 2.5. if the map $\varphi_{j+1}$ is isomorphic on the Ell-group, then so is the map $\varphi_{j}$.

The proof of Theorem 2.5 is complete.

\section{Application to the Monthubert-Nistor indeX}

Let us discuss the relationship with the problems considered by Monthubert and Nistor [15]. In the notation of the present paper, for the case of manifolds with embedded corners they considered the short exact sequence

$$
0 \rightarrow J \longrightarrow \Psi(M) \stackrel{\sigma_{0}}{\longrightarrow} C\left(S^{*} M\right) \rightarrow 0,
$$

where $\sigma_{0}$ is the interior symbol map, and the ideal $J$ consists of operators with zero interior symbol. They studied the boundary map corresponding to this sequence:

$$
\delta: K_{*}\left(C\left(S^{*} M\right)\right) \longrightarrow K_{*+1}(J) .
$$

For a closed manifold $J$ is the ideal of compact operators (hence $K_{*}(J) \simeq \mathbb{Z}$ ) and the boundary map coincides with the analytic index. Moreover, Monthubert and Nistor showed that in the general case this map has an important topological meaning: it gives the obstruction to the existence of an invertible operator with a given interior symbol. For these reasons, Monthubert and Nistor call this map the analytic index of manifolds with corners.

We claim that the classification theorem readily implies a $K$-homology criterion for the vanishing of the analytic index. Indeed, consider the diagram

$$
\begin{aligned}
& K_{*+1}\left(M^{\#}\right) \quad \longrightarrow \quad K_{*+1}\left(M_{0}\right) \stackrel{\partial}{\longrightarrow} K_{*}\left(M^{\#} \backslash M_{0}\right) \\
& \varphi_{1} \uparrow \quad \uparrow \varphi_{k+1} \\
& K_{*}(\Psi(M)) \longrightarrow K_{*}\left(C\left(S^{*} M\right)\right) \stackrel{\delta}{\longrightarrow} K_{*+1}(J),
\end{aligned}
$$

where the lower row is the sequence induced by the short exact sequence (6.1) and the upper row is the exact sequence of the pair $M^{\#} \backslash M_{0} \subset M^{\#}$ in $K$-homology. The maps $\varphi_{1}$ and $\varphi_{k+1}$ are induced by quantization of elliptic symbols on $M^{\#}$ and $M_{0}$ correspondingly (cf. (3.7)). The diagram is obviously commutative.

From the exactness of the sequences and the obvious commutativity of the diagram, we obtain the following assertion. Let us assume for simplicity that $M$ has no connected components with empty boundary. 
Proposition 6.1. The analytic index $\delta(x) \in K_{*+1}(J)$ of $x \in K_{*}\left(C\left(S^{*} M\right)\right)$ vanishes if and only if $\partial \varphi_{k+1}(x)=0$.

Proof. 1. There are splittings (cf. (3.8))

$K_{*}(\Psi(M)) \simeq \widetilde{\mathrm{Ell}}_{*+1}(M) \oplus K_{*}(C(M)), \quad K_{*}\left(C\left(S^{*} M\right)\right) \simeq \mathrm{Ell}_{*+1}\left(M_{0}\right) \oplus K_{*}(C(M))$, where $\widetilde{\text { Ell }}$ is the reduced Ell-group generated by operators of index zero. Moreover, the direct summands $K_{*}(C(M))$ can be cancelled in (6.2). This does not affect the boundary map. Hence, we obtain the commutative diagram

$$
\begin{array}{clcl}
\widetilde{K}_{*+1}\left(M^{\#}\right) & \longrightarrow & K_{*+1}\left(M_{0}\right) & \stackrel{\partial}{\longrightarrow} \widetilde{K}_{*}\left(M^{\#} \backslash M_{0}\right) \\
\varphi_{1} \uparrow & & & \\
K_{*}(\Psi(M)) / K_{*}(C(M)) & \longrightarrow & K_{*}\left(C\left(S^{*} M\right)\right) / K_{*}(C(M)) & \stackrel{\delta}{\longrightarrow} K_{*+1}(J),
\end{array}
$$

where $\widetilde{K}_{*}$ is the reduced $K$-homology group generated by operators of index zero.

3. By the classification theorem, the quantization maps $\varphi$ in (6.3) induce isomorphisms. Hence, the commutativity of the diagram readily shows that vanishing of $\delta$ is equivalent to the vanishing of the boundary map $\partial$ in $K$-homology.

The reader can readily rewrite this formula in a more explicit form as a condition on the interior symbol $\sigma_{0}$. There is also a cohomological form of this condition. Needless to say, the cohomological formula is only valid modulo torsion.

Remark. One actually has the group isomorphism

$$
K_{*}(J) \stackrel{\simeq}{\longrightarrow} \widetilde{K}_{*}\left(M^{\#} \backslash M_{0}\right)
$$

determined by quantization of operators with zero interior symbol. (One can readily obtain this isomorphism by reproducing the proof of our classification theorem. In the proof, only the inductive assumption is changed: now for $j=k+1$ we claim that $0=0$.)

\section{Appendix A. Higher Relative Index Theorem}

Consider the map (see Sec. 4.3)

$$
\varphi_{0}: K_{j}\left(C_{0}\left(\mathbb{R}^{j}\right)\right) \rightarrow K_{j-1}\left(\Delta_{j-1}^{\circ}\right)
$$

induced by the map taking a symbol $\sigma(\xi), \xi \in \mathbb{R}^{j}$, to the corresponding translationinvariant operator

$$
\widehat{\sigma}: L^{2}\left(\mathbb{R}^{j}\right) \longrightarrow L^{2}\left(\mathbb{R}^{j}\right) .
$$

Here the space $L^{2}\left(\mathbb{R}^{j}\right)$ is equipped with the following module structure over the algebra $C_{0}\left(\Delta_{j-1}^{\circ}\right)$ of functions on the interior of the simplex: a function $f \in C_{0}\left(\Delta_{j-1}^{\circ}\right)$ is viewed as a radially constant function equal to zero outside the positive quadrant $\mathbb{R}_{+}^{j}$.

Proposition A.1. For the index pairing of the element $\varphi_{0}[\sigma] \in K_{j-1}\left(\Delta_{j-1}^{\circ}\right)$ with an arbitrary element

$$
[a] \in K_{j-1}\left(C_{0}\left(\Delta_{j-1}^{\circ}\right)\right) \simeq \widetilde{K}^{j-1}\left(\mathbb{S}^{j-1}\right),
$$

where $\widetilde{K}$ is the reduced $K$-group, one has the formula

$$
\left\langle\varphi_{0}[\sigma], a\right\rangle=\operatorname{ind}_{t}([\sigma] \times[a])
$$


where the product $[\sigma] \times[a]$ is defined as the composition

$$
K^{j}\left(\mathbb{R}^{j}\right) \times \widetilde{K}^{j-1}\left(\mathbb{S}^{j-1}\right) \rightarrow K^{1}\left(S^{*} \mathbb{R}^{j}\right) \rightarrow K^{0}\left(T^{*} \mathbb{R}^{j}\right),
$$

and $\operatorname{ind}_{t}: K^{0}\left(T^{*} \mathbb{R}^{j}\right) \rightarrow \mathbb{Z}$ is the topological Atiyah-Singer index for $\mathbb{R}^{j}$.

Remark. For $j=1$, this assertion is reduced to the relative index theorem for operators on manifolds with conical points. We mean the formula for the difference of indices of operators with equal interior symbols, or, equivalently, for the index of elliptic operators of the form $1+G$

$$
\operatorname{ind}(1+G)=w(1+g),
$$

where the interior symbol of $G$ is zero and $w(1+g)$ is the winding number of the conormal symbol $1+g$. Hence the index formula (A.2) in the general case can be referred to as the higher relative index formula.

Proof. To be definite, we consider the case of even $j$. (The odd case can be considered in a similar way.)

1. The element

$$
[\sigma] \in K_{0}\left(C_{0}\left(\mathbb{R}^{j}\right)\right)
$$

is determined by some projection-valued function $p(x)$ on $\mathbb{R}^{j}$ equal to the diagonal projection $\operatorname{diag}(1,0)$ at infinity. Conversely, the element

$$
[a] \in K_{j-1}\left(C_{0}\left(\Delta_{j-1}^{\circ}\right)\right) \simeq \widetilde{K}^{j-1}\left(\mathbb{S}^{j-1}\right)
$$

is determined by some invertible function $a(x)$ on the sphere $\mathbb{S}^{j-1}$. To simplify the notation, we assume that this is a scalar function. The matrix case can be considered in a similar way.

2. In this notation, the index pairing $\left\langle\varphi_{0}[\sigma], a\right\rangle$ is by definition equal to the index of the Toeplitz operator (e.g., see [5])

$$
\widehat{p} a: \operatorname{Im} \widehat{p} \longrightarrow \operatorname{Im} \hat{p}
$$

where $\widehat{p}: L^{2}\left(\mathbb{R}^{j}\right) \longrightarrow L^{2}\left(\mathbb{R}^{j}\right)$ - is the projection determined by the symbol $p$, as in A.1.

3. To compute the index of the operator A.3. we make the Fourier transform. Then the operator $\widehat{p}$ becomes the projection $p(x)$, and the space $\operatorname{Im} \widehat{p}$ becomes the space of sections of the bundle given by the range of $p(x)$. Conversely, the operator of multiplication by $a$ passes into a translation-invariant $\psi \mathrm{DO}$ in $\mathbb{R}^{j}$ with principal symbol $a=a(\xi)$. Hence we obtain

$$
\left\langle\varphi_{0}[\sigma], a\right\rangle=\operatorname{ind}(p \widehat{a}: \operatorname{Im} p \longrightarrow \operatorname{Im} p) .
$$

4. The last operator coincides at infinity with a direct sum of the invertible operator $\widehat{a}$ acting on functions. By the index locality property, the difference of their indices is given by the Atiyah-Singer formula, which gives the desired expression A.2.

Let us rewrite this index formula in the equivalent form used in the main text.

Corollary A.2. The following triangle commutes:

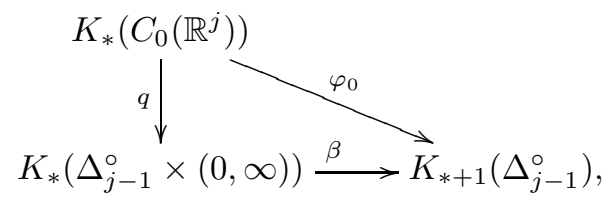


where $\beta$ is the Bott periodicity isomorphism and

$$
q: K_{*}\left(C_{0}\left(\mathbb{R}^{j}\right)\right)=K^{*}\left(T^{*} \mathbb{R}_{+}^{j}\right) \longrightarrow K_{*}\left(\Delta_{j-1}^{\circ} \times(0, \infty)\right)
$$

is the standard pseudodifferential quantization in $\mathbb{R}_{+}^{j}=\Delta_{j-1}^{\circ} \times(0, \infty)$.

Proof. All groups in the triangle A.5 are isomorphic to $\mathbb{Z}$ and have natural Bott generators. It is known that the maps $q$ and $\beta$ take Bott elements to Bott elements. Hence to verify the commutativity of the diagram it suffices to verify this property for $\varphi_{0}$. But this readily follows from the index formula (A.2).

\section{REFERENCES}

[1] M. F. Atiyah, Global theory of elliptic operators, Proc. of the Int. Symposium on Functional Analysis (Tokyo), University of Tokyo Press, 1969, pp. 21-30.

[2] B. Blackadar, $K$-theory for operator algebras, Mathematical Sciences Research Institute Publications, no. 5, Cambridge University Press, 1998, Second edition.

[3] U. Bunke, Index theory, eta forms, and Deligne cohomology, arXiv: math.DG/0201112

[4] A. Connes, Noncommutative geometry, Academic Press Inc., San Diego, CA, 1994.

[5] N. Higson and J. Roe, Analytic K-homology, Oxford University Press, Oxford, 2000.

[6] G. Kasparov, Equivariant KK-theory and the Novikov conjecture, Inv. Math. 91 (1988), no. $1,147-201$

[7] T. Krainer, Elliptic boundary problems on manifolds with polycylindrical ends, Preprint, arXiv: math.AP/0508516 2005.

[8] R. Lauter and S. Moroianu, The index of cusp operators on manifolds with corners, Ann. Global Anal. Geom. 21 (2002), no. 1, 31-49.

[9] P.-Y. Le Gall and B. Monthubert, $K$-theory of the indicial algebra of a manifold with corners, $K$-Theory 23 (2001), no. 2, 105-113.

[10] P. Loya, The index of b-pseudodifferential operators on manifolds with corners, Ann. Global Anal. Geom. 27 (2005), no. 2, 101-133.

[11] G. Luke, Pseudodifferential operators on Hilbert bundles, J. Diff. Equations 12 (1972), 566589

[12] R. Melrose and V. Nistor, K-theory of $\mathbf{C}^{*}$-algebras of b-pseudodifferential operators, Geom. Funct. Anal. 8 (1998), no. 1, 88-122.

[13] R. Melrose and P. Piazza, Analytic K-theory on manifolds with corners, Adv. in Math. 92 (1992), no. 1, 1-26.

[14] B. Monthubert, Groupoids and pseudodifferential calculus on manifolds with corners, J. Funct. Anal. 199 (2003), no. 1, 243-286.

[15] B. Monthubert and V. Nistor, A topological index theorem for manifolds with corners, arxiv: math.KT/0507601 2005.

[16] V. Nistor, An index theorem for gauge-invariant families: The case of solvable groups, Acta Math. Hungarica 99 (2003), no. 2, 155-183.

[17] A. Savin, Elliptic Operators on Singular Manifolds and K-homology, K-theory 34 (2005), no. 1, 71-98.

[18] V. E. Nazaikinskii, A. Yu. Savin, and B. Yu. Sternin, On the homotopy classification of elliptic operators on stratified manifolds, arXiv: math.KT/0608332 2005.

[19] V. E. Nazaikinskii, A. Yu. Savin, and B. Yu. Sternin, Elliptic Theory on Manifolds with Corners: I. Dual Manifolds and Pseudodifferential Operators, arXiv: math.OA/0608353 2006.

Institute for Problems in Mechanics, Russian Academy of Sciences, PR. Vernadskogo 101-1, 119526 Moscow, Russia

E-mail address: nazaikinskii@yandex.ru

Independent University of Moscow, Bol'shoi Vlas'evskil Per. 11, 119002 Moscow, Russia

E-mail address: antonsavin@mail.ru

E-mail address: sternin@mail.ru 